
patient is at home http://ow.ly/Snx69

Simon Tiberi ${ }^{1,7}$, Lia D’Ambrosio ${ }^{2,3,7}$, Saverio De Lorenzo ${ }^{4,7}$, Pietro Viggiani ${ }^{4}$, Rosella Centis ${ }^{3}$, Giovanni Sotgiu ${ }^{5}$, Jan Wilem C. Alffenaar ${ }^{6}$ and Giovanni Battista Migliori $^{3}$

${ }^{1}$ Division of Infection, Barts Healthcare NHS Trust, London, UK. ${ }^{2}$ Public Health Consulting Group, Lugano, Switzerland. ${ }^{3}$ WHO Collaborating Centre for TB and Lung Diseases, Fondazione S. Maugeri, Tradate, Italy. ${ }^{4}$ Eugenio Morelli MDR-TB Reference Hospital, AOVV, Sondalo, Italy. ${ }^{5}$ Clinical Epidemiology and Medical Statistics Unit, Dept of Biomedical Sciences, University of Sassari - Research, Medical Education and Professional Development Unit, AOU Sassari, Sassari, Italy. ${ }^{6}$ University of Groningen, University Medical Center Groningen, Dept of Clinical Pharmacy and Pharmacology, Groningen, The Netherlands. ${ }^{7}$ These authors contributed equally.

Correspondence: Giovanni Battista Migliori, WHO Collaborating Centre for TB and Lung Diseases, Fondazione S. Maugeri, Via Roncaccio 16, Tradate, Varese 21049, Italy. E-mail: giovannibattista.migliori@fsm.it

Received: Aug 032015 | Accepted after revision: Aug 262015 | First published online: Nov 192015

Conflict of interest: None declared.

\title{
References
}

1 World Health Organization. Global tuberculosis report 2014. WHO/HTM/TB2014.08. Geneva, World Health Organization, 2014.

2 Falzon D, Jaramillo E, Schünemann HJ, et al. WHO guidelines for the programmatic management of drug-resistant tuberculosis: 2011 update. Eur Respir J 2011; 38: 516-528.

3 Falzon D, Gandhi N, Migliori GB, et al. Resistance to fluoroquinolones and second-line injectable drugs: impact on multidrug-resistant TB outcomes. Eur Respir J 2013; 42: 156-168.

4 Migliori GB, Sotgiu G, Gandhi NR, et al. Drug resistance beyond extensively drug-resistant tuberculosis: individual patient data meta-analysis. Eur Respir J 2013; 42: 169-179.

5 Blasi F, Dara M, van der Werf MJ, et al. Supporting TB clinicians managing difficult cases: the ERS/WHO Consilium. Eur Respir J 2013; 41: 491-494.

6 Diel R, Vandeputte J, de Vries G, et al. Costs of tuberculosis disease in the European Union: a systematic analysis and cost calculation. Eur Respir J 2014; 43: 554-565.

7 De Lorenzo S, Alffenaar JW, Sotgiu G, et al. Efficacy and safety of meropenem-clavulanate added to linezolid-containing regimens in the treatment of MDR-/XDR-TB. Eur Respir J 2013; 41: 1386-1392.

8 Cordillot M, Dubée V, Triboulet S, et al. In vitro cross-linking of Mycobacterium tuberculosis peptidoglycan by L,D-transpeptidases and inactivation of these enzymes by carbapenems. Antimicrob Agents Chemother 2013; 57: 5940-5945.

9 Tremblay LW, Fan F, Blanchard JS. Biochemical and structural characterization of Mycobacterium tuberculosis beta-lactamase with the carbapenems ertapenem and doripenem. Biochemistry 2010; 49: 3766-3773.

10 De Lorenzo S, Centis R, D’Ambrosio L, et al. On linezolid efficacy and tolerability. Eur Respir J 2012; 39: 770-772.

11 Sotgiu G, Centis R, D'Ambrosio L, et al. Efficacy, safety and tolerability of linezolid containing regimens in treating MDR-TB and XDR-TB: systematic review and meta-analysis. Eur Respir J 2012; 40: 1430-1442.

12 Srivastava S, Peloquin CA, Sotgiu G, et al. Therapeutic drug management: is it the future of multidrug-resistant tuberculosis treatment? Eur Respir J 2013; 42: 1449-1453.

13 Tiberi S, De Lorenzo S, Centis R, et al. Bedaquiline in MDR/XDR-TB cases: first experience on compassionate use. Eur Respir J 2014; 43: 289-292.

14 Esposito S, D'Ambrosio L, Tadolini M, et al. ERS/WHO Tuberculosis Consilium assistance with extensively drug-resistant tuberculosis management in a child: case study of compassionate delamanid use. Eur Respir J 2014; 44: 811-815.

15 Sotgiu G, D'Ambrosio L, Centis R, et al. TB and M/XDR-TB infection control in European TB reference centres: the Achilles' heel? Eur Respir J 2011; 38: 1221-1223.

\section{Restrictive chronic lung function decline after haematopoietic stem cell transplantation}

To the Editor:

Until recently, it was generally thought that bronchiolitis obliterans syndrome (BOS), which is a type of obstructive lung disease with a dismal outcome, was the sole form of chronic lung allograft dysfunction 
(CLAD) occurring after lung transplantation. However, recent publications reported that some patients experience a restrictive form of chronic lung complication that has been termed restrictive allograft syndrome (RAS) [1]. Approximately one-third of patients with CLAD after lung transplantation can be classified as having RAS $[2,3]$. This distinct form of CLAD is considered to be an important entity and has features that are distinct from those of BOS. First and foremost, the mortality of RAS is even greater than that from BOS: a previous study reported that the survival of patients with RAS was significantly poorer than that of patients with BOS [3]. Secondly, imaging of BOS on computed tomography (CT) has revealed air trapping, tree-in-bud opacities and peribronchial infiltrates, whereas RAS presented mainly with alveolar or interstitial changes such as septal thickening or reticulation [4]. Additionally, RAS has been histologically characterised by diffuse alveolar damage and extensive fibrosis in the alveolar interstitium and interlobular septa [2].

Lung transplantation is not the only cause of BOS; that is, BOS can also develop after haematopoietic stem cell transplantation (HSCT) [5]. We speculated therefore that a restrictive form of chronic lung complication might also occur after HSCT and aimed to investigate whether a chronic, restrictive lung complication can indeed develop in HSCT recipients and, if so, to identify its characteristics.

We recruited study subjects who underwent HSCT at Asan Medical Center, a 2700-bed referral hospital in Seoul, South Korea, between December 1993 and November 2013. In total, 1352 patients were initially recruited into our study, leading to a final number of participants of 1187 (figure 1). The medical records of these patients were analysed retrospectively in June 2014. Our current study protocol was approved by the Institutional Review Board of Asan Medical Center.

Chronic lung function decline (CLFD) is defined as a reduction of $>20 \%$ compared with the corresponding baseline values at the time of HSCT of the average of the two best pre-bronchodilator forced expiratory volumes in $1 \mathrm{~s}(\mathrm{FEV} 1)$ and/or forced vital capacity (FVC) measurements taken $\geqslant 3$ weeks apart. In all patients, BOS and restrictive CLFD (R-CLFD) were diagnosed on the basis of pulmonary function test results. The criteria for defining BOS were 1) the presence of CLFD, 2) a FEV1/FVC ratio $<0.7$, and 3) the absence of an identifiable cause of lung function decline, such as infection. The criteria for R-CLFD were 1) the presence of CLFD, 2) a FEV1/FVC ratio $\geqslant 0.7$ and 3) the absence of an identifiable cause.

The mean age of the 1187 patients with HSCT was 39.0 years and men predominated (56.3\%, 668 out of 1187 patients). Acute leukaemia was the most common indication for HSCT (64.3\%, 763 out of 1187 patients).

After excluding identifiable causes, 88 patients were diagnosed with CLFD during a median follow-up period of 42.5 months (interquartile range (IQR) 19.7-60.0 months) after HSCT (figure 1). Of these, BOS developed in $82(93.2 \%)$ patients at a median of 12.3 months (IQR 8.8-21.1 months) after HSCT. At a median of 30.3 months (IQR 15.2-51.5 months) after HSCT, another six (6.8\%) patients were diagnosed

Patients with haematologic malignancy who received HSCT from December 1993 to November 2013 ( $n=1352)$

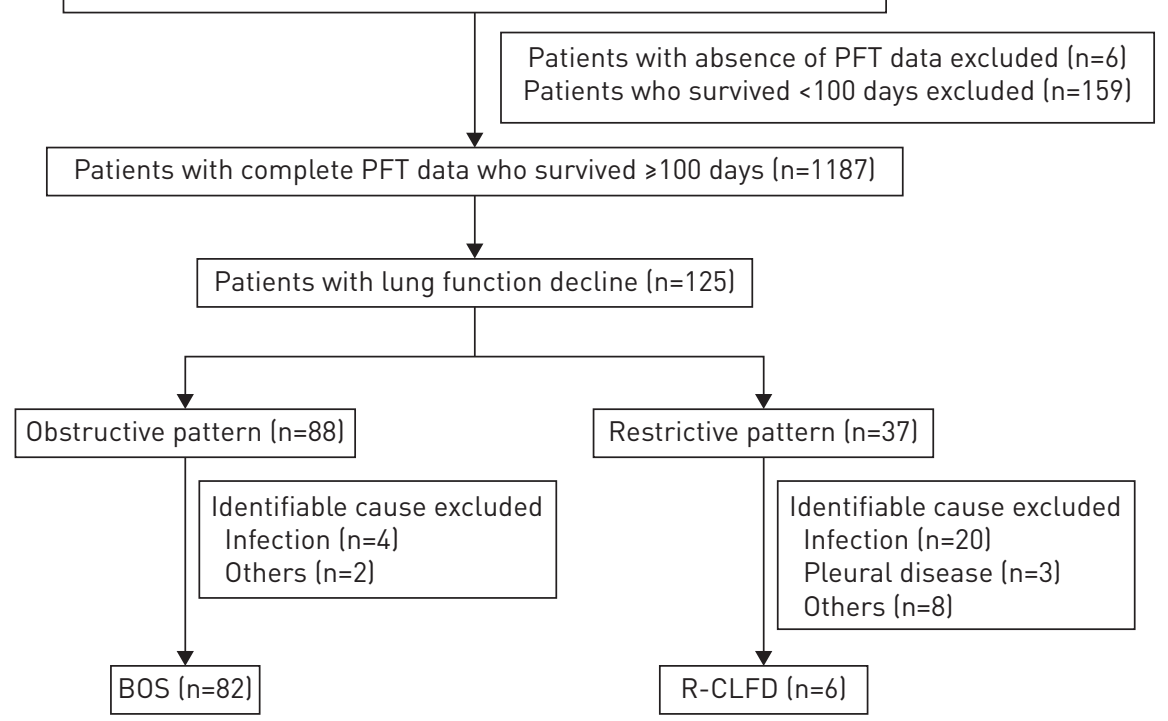

FIGURE 1 Screening and enrolment of study patients. HSCT: haematopoietic stem cell transplantation; PFT: pulmonary function test; BOS: bronchiolitis obliterans syndrome; R-CLFD: restrictive chronic lung function decline. 
with R-CLFD. Baseline characteristics were comparable between the six patients with R-CLFD and the 82 BOS cases. All patients with BOS had evidence of chronic graft-versus-host disease (GVHD) at another site, such as the skin or liver.

Excluding the three patients who received a lung transplant, the survival of the remaining 79 patients with BOS was compared with that of the six patients with R-CLFD. A total of 26 (32.9\%) BOS patients died, mostly from infection, and the median survival of the 79 patients with BOS was 20.0 months (IQR 5.7-40.3 months) from diagnosis. In contrast, the median survival time of the six R-CLFD patients was 42.3 months (IQR 29.1-100.2 months) from diagnosis ( $\mathrm{p}<0.001$ compared with BOS) and one $(16.7 \%)$ patient died of septic shock due to pneumonia.

For those BOS patients who were investigated by chest CT, the findings were consistent with their diagnosis: namely, mosaic attenuation, hyperlucent lung parenchyma, bronchial wall thickening and centrilobular lung nodules. In contrast, radiological examination of R-CLFD showed slowly progressive fibrotic changes mainly involving alveolar units. These changes usually started with ground-glass opacities or consolidation of the peripheral lung and were followed by gradually increased interstitial reticulation, before finally progressing to volume loss and architectural distortion with traction bronchiectasis.

To the best of our knowledge, our current study is the first to report chronic lung complications of a restrictive type in patients who have received HSCT. The most important finding of our present analyses was that some patients presented with a restrictive form of CLFD after HSCT.

Our CT findings were consistent with those previously reported for RAS after lung transplantation [6], and our patients with RAS and R-CLFD showed the same restrictive pulmonary physiology. However, we found that some features of R-CLFD and BOS after HSCT differed from those of RAS and BOS after lung transplantation. Above all, the incidence of R-CLFD was only $6.8 \%$ in the 88 patients in our CLFD group that combined BOS and R-CLFD. Besides this, the survival of patients with R-CLFD was significantly better than that of patients with BOS. These features contrast with those of R-CLAD after lung transplantation.

BOS is associated with constrictive bronchiolitis that targets conducting airways while sparing terminal bronchioles and the alveolar surface [7], whereas the lung changes mainly developed in alveolar units in $\mathrm{R}$-CLFD in our present study. This disparity in anatomical involvement sites suggests that the pathobiological mechanisms of R-CLFD after HSCT are different from those of BOS, which is considered to be a manifestation of chronic GVHD. ToDD et al. [3] have reported that patients with R-CLAD after lung transplantation were more likely to have newly detected human leukocyte antigen (HLA) antibodies than those with BOS, which may have humoral responses that directly target alveolar antigens. In this context, we believe that the reason for the different incidence of restrictive chronic lung complications between post-HSCT and post-lung transplantation patients might be partly explained by the presence of anti-HLA antibodies; that is, in contrast to lung transplantation, where HLA matching is not usually a concern, HLA matching is strongly recommended in HSCT. Indeed, in the 1187 patients who were included in our current analysis, 769 (64.8\%) patients and 350 (29.5\%) patients received matched sibling and matched unrelated HSCT, respectively.

We excluded patients with infection as the identifiable cause before a CLFD diagnosis. In these patients, the infection had been appropriately treated and lung function subsequently recovered in most cases. None of the six patients with R-CLFD experienced symptoms suggestive of infection when early radiological changes such as ground-glass opacities or consolidation occurred.

In conclusion, we here report that a restrictive type of lung function decline can develop as a chronic lung complication of HSCT in a subset of patients. This distinct type of CLFD seems to have features that differ from those of BOS after HSCT in terms of radiological findings, incidence and survival.

@ERSpublications

Some patients present with restrictive chronic lung function decline after haematopoietic stem cell transplantation http://ow.ly/RpsAx

Ji-Young Yang ${ }^{1}$, Sang Young $\mathrm{Oh}^{2}$, Jin Woo Song ${ }^{1}$, Tae Sun Shim ${ }^{1}$, Sei Won Lee ${ }^{1}$, Jae Seung Lee', Dae-Young Kim ${ }^{3}$, Je-Hwan Lee ${ }^{3}$, Jung-Hee Lee ${ }^{3}$, Kyoo-Hyung Lee ${ }^{3}$ and Kyung-Wook Jo ${ }^{1}$

${ }^{1}$ Division of Pulmonary and Critical Care Medicine, University of Ulsan College of Medicine, Asan Medical Center, Seoul, South Korea. ${ }^{2}$ Dept of Radiology, University of Ulsan College of Medicine, Asan Medical Center, Seoul, South Korea. ${ }^{3}$ Division of Hematology, University of Ulsan College of Medicine, Asan Medical Center, Seoul, South Korea.

Correspondence: Kyung-Wook Jo, Division of Pulmonary and Critical Care Medicine, University of Ulsan College of Medicine, Asan Medical Center, 388-1 Pungnap-dong, Songpa-gu, Seoul 138-736, South Korea.

E-mail: heathcliff6800@hanmail.net

Received: Feb 012015 | Accepted after revision: July 102015 | First published online: Sept 172015

Conflict of interest: None declared. 


\title{
References
}

1 Verleden GM, Raghu G, Meyer KC, et al. A new classification system for chronic lung allograft dysfunction. J Heart Lung Transplant 2014; 33: 127-133.

2 Sato M, Waddell TK, Wagnetz U, et al. Restrictive allograft syndrome (RAS): a novel form of chronic lung allograft dysfunction. J Heart Lung Transplant 2011; 30: 735-742.

3 Todd JL, Jain R, Pavlisko EN, et al. Impact of forced vital capacity loss on survival after the onset of chronic lung allograft dysfunction. Am J Respir Crit Care Med 2014; 189: 159-166.

4 Verleden SE, de Jong PA, Ruttens D, et al. Functional and computed tomographic evolution and survival of restrictive allograft syndrome after lung transplantation. J Heart Lung Transplant 2014; 33: 270-277.

5 Barker AF, Bergeron A, Rom WN, et al. Obliterative bronchiolitis. N Engl J Med 2014; 370: 1820-1828.

6 Sato M, Hwang DM, Waddell TK, et al. Progression pattern of restrictive allograft syndrome after lung transplantation. J Heart Lung Transplant 2013; 32: 23-30.

7 Verleden SE, Vasilescu DM, Willems S, et al. The site and nature of airway obstruction after lung transplantation. Am J Respir Crit Care Med 2014; 189: 292-300

\section{Detection of pepsin in sputum: a rapid and objective measure of airways reflux}

\author{
To the Editor:
}

The diagnostic test, Peptest (RD Biomed Ltd, Cottingham, UK), detects pepsin in expectorated saliva and is established as a simple, noninvasive measure of reflux of gastric contents. It has been used to detect pepsin A in patients with both gastro-oesophageal reflux disease (GORD) and extra-oesophageal reflux into the laryngopharynx and airways [1-5].

Reflux has a substantial, but as yet undefined, component in the aetiology of cough hypersensitivity syndrome [6]. Currently evaluation is by the subjective Hull Airways Reflux Questionnaire (HARQ) [7] or objective, but costly and invasive, measures such as 24-h pH-metry, impedance [8], and high resolution manometry $[8,9]$. These current diagnostic pathways have their limitations in detecting low levels of airways reflux that may be sufficient to cause chronic cough. We hypothesised that Peptest could provide simple objective confirmation of airways reflux in unselected patients with chronic cough.

Peptest was used in routine clinical practice in out-patients attending the Hull Cough Clinic at Castle Hill Hospital (Hull, UK), a secondary and tertiary referral centre. Verbal consent was obtained at the time of attendance. Chronic cough was defined as cough lasting $>8$ weeks.

Patients were instructed to provide three expectorated saliva/sputum samples into sample collection tubes (containing $0.01 \mathrm{M}$ citric acid) during daily activities and immediately after three spontaneous coughing episodes. Sample collection was optimised by providing patient leaflets and a video. The presence and concentration of pepsin was measured using Peptest by trained analysts and with a lateral flow-test reader calibrated with known concentrations of pepsin A standard. The lower limit of detection is $16 \mathrm{ng} \cdot \mathrm{mL}^{-1}$ and upper limit of quantification was $500 \mathrm{ng} \cdot \mathrm{mL}^{-1}$. Peptest is specific for pepsin $\mathrm{A}$ (isoforms $1,3 \mathrm{a}, 3 \mathrm{~b}$ and $3 \mathrm{c}$ ) and does not detect pepsin C/Gastricsin (isoform 5) putatively suggested to be expressed in the lungs [10, 11]. As pepsin concentrations do not follow a normal distribution a non-parametric statistical analysis was performed.

We have tested 93 (55 female) chronic cough patients mean \pm SD age 58.4 \pm 13.8 years between August 2014 and December 2014. Smoking status was: smoker $n=5$, nonsmoker $n=55$, ex-smoker $n=24$ and unknown $n=9$. The mean \pm SD HARQ score (upper limit of normal 13) was 31.9 \pm 13.1 and cough duration was $5.6 \pm 7.0$ years. Over a period of 4 months the 93 patients provided 262 evaluable samples for testing. 80 patients had at least one pepsin positive sample $(86.0 \%)$. Pepsin concentrations ranged from 0 to $500 \mathrm{ng} \cdot \mathrm{mL}^{-1}$ with a median (interquartile range (IQR)) of $31 \mathrm{ng} \cdot \mathrm{mL}^{-1}(0-113.5) \mathrm{ng} \cdot \mathrm{mL}^{-1}$.

We previously [1] used a similar triple sampling strategy in a thoroughly investigated normal asymptomatic healthy volunteer population. The absence of gastro-oesophageal reflux was confirmed by $\mathrm{pH}$-impedance testing. In contrast to this chronic cough study these control samples were provided first thing in the morning, $1 \mathrm{~h}$ after lunch and $1 \mathrm{~h}$ after the evening meal. Of the 87 control subjects only 33 were found to have at least one positive sample (37.9\%) but the pepsin concentration in those that were Peptest positive was very low, median (IQR) of $0(0-0)$ ng. $\mathrm{mL}^{-1}$ pepsin, which represents physiological reflux. 